\title{
Comunicação
}

\section{Neoplasias associadas ao carcinoma epidermóide do esôfago}

\author{
C.C. Schirmer, R. Gurski, M.A.A. Castro, G.S.P. Madruga, F.L. Pedroso, C.D.P. Kruel, L. Brentano
}

Grupo de Cirurgia do Esôfago, Estômago e Intestino Delgado (GCEEID), Hospital de Clínicas de Porto Alegre, Universidade F ederal do Rio Grande do Sul, Porto Alegre, RS.

RESUMO - INTRODUÇão. O carcinoma epidermóide de esôfago (CEE) tem uma importante associação com neoplasias do trato aerodigestivo e, provavelmente, compartilham dos mesmos fatores de risco. Além destes, outras neoplasias podem estar associadas com o carcinoma de esôfago.

Овј етіvo. Analisar, retrospectivamente, pacientes com carcinoma epidermóide do esôfago tratados pelo Grupo de Cirurgia do E sôfago, E stômago e Intestino Delgado (GCEEID) do Hospital de Clínicas de Porto Alegre (HCPA), no período de janeiro/ 88 a junho/95, os quais tinham neoplasias associadas ao CEE.

Pacientes e Métodos. Dentre os 261 pacientes estudados, 19 (7,28\%) tinham neoplasia associada ao CEE. Dez pacientes apresentaram tumores sincrônicos e 9, metacrônicos. 0 sexo predominante foi o masculino, com 17 casos. A média de idade fi cou em 62,52 anos no momento do diagnósti co da neoplasia esofágica.

Resultados. Os tumores aerodigestivos, na sua totalidade carcinomas escamosos, representaram o tipo histológico predominante da neoplasia associada em $68,42 \%$ dos casos. $O$ sítio mais freqüen-

\section{NTRODUÇÃO}

Desde a primeira descrição de Billroth sobre a segunda neoplasia primária em indivíduos com câncer, em 1860, existe grande interesse sobre essa propensão natural, principalmente em pessoas com carcinoma de cabeça e pescoço. Esses indivíduos têm alto risco para desenvolver uma segunda neoplasia primária local ou a distância, assim como metástases ${ }^{1}$. Além disso, devido a meIhores resultados dos tratamentos e, conseqüentemente, aumento na sobrevida dos pacientes com câncer, a incidência de neoplasias associadas em diferentes sítios tem aumentado nos últimos anos. A associação existente entre o CEE com tumores aerodigestivos é conheci da e tem sido reportada na literatura mundial ${ }^{2-4}$, sendo o tabaco e o álcool fatores importantes na eti ol ogia desses tumores ${ }^{5-7}$. Além disso, fatores genéticos podem ter papel te da neoplasia aerodigestiva associada foi a árvore respi ratória $(53,8 \%)$, seguido da cavidade oral e or ofaringe ( $23 \%$ ) e laringe ( $23 \%$ ). Dos 19 pacientes, 12 eram tabagistas e nove ingeriam bebidas alcoólicas regularmente. Para o tratamento do CEE, optou-se por cirurgia em seis pacientes. A neoplasia associada foi tratada com cirurgia radical em 11 pacientes e radioterapia em cinco. Surpreendentemente, foram diagnosticados quatro casos (21\%) de adenocarci nomas gástricos associados ao CEE, tratados com cirurgia radical em três pacientes.

Conclusão. Os autores ressaltam a importância do estadiamento criterioso dos pacientes com CEE devido a associação significativa com outras neoplasias, principalmente com tumores aerodigestivos. Alertam para o seguimento desses pacientes e discutem a possibilidade de fatores de risco comuns: fumo e álcool. Nesta casuística, encontrou-se associação importante com neoplasias gástricas.

UNITERMOS: Carcinoma de células escamosas. Neoplasia esofágica. Neoplasias associadas. Neoplasias primárias múltiplas. Tumores aerodigestivos. rel evante, devido a associação de casos de tumores do trato aerodigestivo superior com história familiar positiva para neoplasias de cabeça e pescoço e de esôfago ${ }^{8-10}$. Outras associações, como as que envol vem fatores de risco físicos (calor) e químicos (nitrosaminas), ainda são objeto de pesquisa experimental. Devido a essa freqüente associação, indica-se o estadi amento e o acompanhamento criteriosos dos pacientes com CEE. No presente estudo, são descritos 19 casos de tumores malignos associados ao CEE, suas circunstâncias diagnósticas e o tratamento realizado.

\section{PACIENTES E MÉTODOS}

No período de janeiro de 1988 a junho de 1995, foram avaliados e tratados 261 pacientes portadores de CCE pelo GCEEID do Hospital de Clínicas de Porto Alegre. Em 19 casos (7,28\%), constatou-se 
Tabela 1 - Relação dos tumores associados ao CEE

\begin{tabular}{|c|c|c|c|}
\hline Caso* & Anterior** & Simultâneo & Posterior ${ }^{\star *}$ \\
\hline 1. LS, f, 70 anos & $\begin{array}{l}\text { Tiróide - Neoplasia folicular } \\
4 \text { meses }\end{array}$ & & \\
\hline 2. OJ, m, 53 anos & & Brônquio esquerdo - Ca epidermóide & \\
\hline 3. IS, $\mathrm{m}, 48$ anos & & Brônquio esquerdo - Ca epidermóide & \\
\hline 4. DL, $\mathrm{m}, 62$ anos & $\begin{array}{l}\text { Pulmão - Ca epidermóide } \\
48 \text { meses }\end{array}$ & & \\
\hline 5. SS, $\mathrm{m}, 63$ anos & $\begin{array}{l}\text { Laringe - Ca epidermóide } \\
48 \text { meses }\end{array}$ & & \\
\hline 6. JM, m, 58 anos & & & $\begin{array}{l}\text { Mucosa oral - Ca epidermóide } \\
25 \text { meses }\end{array}$ \\
\hline 7. TP, $\mathrm{m}, 65$ anos & & Estômago - Adenocarcinoma & \\
\hline 8. GL, $\mathrm{m}, 62$ anos & $\begin{array}{l}\text { Tumor de base de língua } \\
84 \text { meses }\end{array}$ & & \\
\hline 9. LF, $\mathrm{m}, 58$ anos & & Pulmão - Ca epidermóide & \\
\hline 10. LM, $\mathrm{m}, 65$ anos & $\begin{array}{l}\text { Laringe - Ca epidermóide } \\
15 \text { meses }\end{array}$ & & \\
\hline 11. TS, m, 58 anos & & Estômago - Adenocarcinoma & \\
\hline 12. DO, f, 53 anos & & Brônquio principal direito - Ca epidermóide & \\
\hline 13. SS, $\mathrm{m}, 73$ anos & $\begin{array}{l}\text { Laringe - Ca epidermóide } \\
149 \text { meses }\end{array}$ & & \\
\hline 14. BP, $\mathrm{m}, 69$ anos & $\begin{array}{l}\text { Próstata - Adenocarcinoma } \\
72 \text { meses }\end{array}$ & & \\
\hline 15. NC, $\mathrm{m}, 65$ anos & & Pulmão - Ca epidermóide & \\
\hline 16. $A E, m, 65$ anos & & Pulmão - Ca epidermóide & \\
\hline 17. NP, $m, 44$ anos & $\begin{array}{l}\text { Orofaringe - Ca epidermóide } \\
15 \text { meses }\end{array}$ & & \\
\hline $\begin{array}{l}\text { 18. JJ, m, } 77 \text { anos } \\
\text { 19. EK, m, } 69 \text { anos }\end{array}$ & & $\begin{array}{l}\text { Estômago - Adenocarcinoma } \\
\text { Estômago - Adenocarcinoma }\end{array}$ & \\
\hline
\end{tabular}

presença de neoplasia associada ao CEE. Esses tumores associados ocorreram em outros órgãos, descartando-se a possibilidade de metástases ou invasão por contigüidade do CEE. Esses casos foram estudados retrospectivamente, sendo considerado sincrônico os que o diagnóstico da neoplasia associada não tenha ul trapassado o período de três meses do diagnóstico do carcinoma esofágico, e metacrônico as demais. Dos 19 pacientes com neoplasia associada ao CEE, 17 eram do sexo masculino e dois do feminino, com idades entre 48 e 77 anos, com média de 62,52 no momento do diagnóstico da neoplasia esofágica. Destes, dez eram tumores sincrônicos e nove, metacrônicos (tabela 1).

O protocolo de estadiamento e acompanhamento dos pacientes com CEE incluem: exame clínico minucioso, estudo radiológico contrastado do trato digestivo superior, endoscopia digestiva alta, avaliação otorrinolaringológica, tomografia axial computadorizada do mediastino, estudo radiológico do tórax, fibrobroncoscopia, ecografia abdominal e, recentemente, a ecoendoscopia. O diagnóstico da neoplasia associada foi em todos os casos confirmado mediante exame anatomo ou citopatológico, con- forme biópsia ou punção aspirativa da lesão.

Os tratamentos realizados para O CEE e para a neoplasia associada nos 19 pacientes, assim como o seguimento desses doentes, estão demonstrados na tabela 2.

Os testes utilizados para o estudo estatístico foram o qui quadrado $\left(\chi^{2}\right)$ e o teste t de Student.

\section{RESULTADOS}

O sítio mais freqüente da neoplasia aerodigestiva associada foi a árvore respiratória com sete casos $(46,7 \%)$, seguida por tumores da cavidade oral e orofaringe, três casos (23\%), e tumores da laringe, três casos (23\%) [tabela 1]. E $m$ todos esses pacientes, o tipo histológi co foi o mesmo da neoplasia esofágica. Quanto aos outros seis pacientes com neoplasias associadas ao CE E e não-aerodigestivas, ocorreram quatro casos (21\%) de adenocarcinoma gástrico, um adenocarcinoma de próstata e um de carcinoma folicular de tiróide. Todos os pacientes da amostra tinham história de tabagismo, sendo 14 deles fumantes e cinco, ex-fumantes. A informação sobre o tempo e quantificação do 


\begin{tabular}{|cll|}
\hline Tabela 2 - Tratamento e seguimento & \\
\hline Caso & \multicolumn{1}{c|}{ CEE } & \multicolumn{1}{c|}{ Neoplasia associada } \\
1 & Radioterapia & Ablação com ${ }^{131}+$ tiroidectomia + T3 \\
2 & Radioterapia & Radioterapia \\
3 & Prótese de Malafaia & Nenhum tratamento \\
4 & Quimioterapia & Pneumonectomia E \\
5 & Radioterapia & Laringectomia fronto-lateral E + traqueostomia \\
6 & Esofagectomia c/ toracotomia & Mandibulectomia E \\
7 & Esofagectomia c/ toracotomia & Gastrectomia \\
8 & Esofagectomia c/ toracotomia & Radioterapia + hemiglossectomia \\
9 & Nenhum tratamento & Nenhum tratamento \\
10 & Prótese de Malafaia & Laringectomia total \\
11 & Nenhum tratamento & Nenhum tratamento \\
12 & Gastrostomia (mal est. geral, caquexia) & Paliativa \\
13 & Prótese de Malafaia p/ vídeo & Laringectomia + traqueostomia \\
14 & Esofagectomia c/ toracotomia & Prostatectomia \\
15 & Radioterapia & Radioterapia \\
16 & Radioterapia & Radioterapia \\
17 & Esofagogastroplastia cervical & Radioterapia \\
18 & Esofagogastrectomia transmediastinal & Esofagogastrectomia transmediastinal \\
19 & Nenhum tratamento & Nenhum tratamento \\
\hline
\end{tabular}

tabagismo não foi coletada dos prontuários, pois não havia padronização dos dados. Em relação a ingesta de bebi das al coólicas, 12 pacientes afirmaram que a faziam regularmente, quatro referiam etilismo no passado e três negavam. O tratamento mais utilizado para a neoplasia associada foi somente cirurgia em nove casos $(47,36 \%)$, segui do de cirurgia e radioterapia em um $(5,26 \%)$, somente radioterapia em quatro (21,05\%), paliativo em quatro (21,05\% - prótese em dois e nenhum tratamento em dois casos) e outro tratamento em um caso $(5,26 \%)$ [tabela 2 ]. O tratamento do carcinoma epidermóide de esôfago foi paliativo em sete casos (36,84\% - prótese em quatro e nenhum tratamento em três casos), somente radioterapia em cinco $(26,3 \%)$, somente cirurgia em seis $(31,57 \%)$ e quimi oterapia em um caso $(5,26 \%)$ [tabela 2 ]. As associações entre tabagi smo, etilismo, sítio da neoplasia associada, idade e sexo não foram estatisticamente significantes $(p>0,05)$ ou não possuíam relevância clínica pelo tamanho da amostra.

\section{DISCUSSÃo}

Billroth, em 1860, descreveu pela primeira vez a existência de um segundo tumor primário em pacientes com neoplasia de cabeça e pescoço. $\mathrm{Nu}$ merosos estudos posteriores demonstraram que mais de $25 \%$ desses doentes desenvolveram uma segunda neoplasia ao longo de suas vidas ${ }^{11}$. Vários outros autores apresentam estudos retrospectivos e prospectivos que demonstram a presença de neoplasias simultâneas ou metacrônicas em pacientes com tumores aerodigestivos. Em estudo re- trospectivo de 1.091 pacientes com neoplasias da cabeça e pescoço, Savary et al. ${ }^{2}$ encontraram 180 pacientes $(16,5 \%)$ com tumores malignos sincrônicos ou metacrônicos múltiplos. Desses, 53\% eram de cabeça e pescoço, $23 \%$ no esôfago e $24 \%$ nos brônquios. Os mesmos autores referem um risco cumulativo, em cinco anos, para um segundo tumor, de $32 \%$, nos pacientes alcoólatras e tabagistas pesados. O risco cumulativo em cinco anos para um terceiro tumor é de $55 \%$ para os pacientes que já tiveram dois tumores. Em outros dois trabal hos prospectivos, foram real izadas panendoscopias em 100 pacientes com câncer de cabeça e pescoço, sendo observada, em 19 deles, a existência de segundo tumor primário simultâneo: dez casos no esôfago e seis no brônquio. Em $89 \%$ dos casos, a segunda neoplasia era um carcinoma in situ ou T1. Savary et al. concluíram que o risco cumulativo em cinco anos de desenvolver a segunda neoplasia em pacientes com tumores aerodigestivos é três vezes maior do que nos não expostos.

Outros autores, como Elias et al.12, apresentaram estudo retrospectivo com números mais impressionantes. Na casuística de 181 pacientes submetidos a cirurgia por carcinoma epidermóide do esôfago, encontraram 56\% (102 pacientes) com neoplasias associadas, dos quais $50 \%$ eram de tumores de cabeça e pescoço. Em 53 pacientes, a neoplasia associada foi sincrônica ao CEE, em 40 foi metacrônica anterior e, em nove, de neoplasia metacrônica posterior. Essa alta incidência de neoplasias associadas deve-se, provavelmente, ao tabagismo e ao al cool ismo, que atuam como fatores de risco comuns ${ }^{12}$. 
Estudo realizado por Morita et al. ${ }^{7}$ sugere que a história familiar de câncer de cabeça e pescoço, assim como de tabagismo pesado e de etilismo, está claramente definida como fatores de risco para a ocorrência de neoplasias associadas ao trato aerodigestivo.

A presença de neoplasias associadas ao CEE, relatada na literatura internacional, varia de $5 \%$ a $20 \%$; na sua maioria tumores aerodigestivos. A explicação para esse fato parece também evidente e está fundamentada nos fatores de risco comuns: tabagismo, al cool ismo e história familiar. A falta de protocolos uniformes no estadiamento das neoplasias originadas de epitélio escamoso no tubo digestivo superior e vias aéreas permite que um grande número de tumores associados não seja diagnosticado.

Nossa experiência no GCEEID-HCPA desde 1988, numa casuística de 261 pacientes com CEE, fundamentados em protocolo extenso e rigidamente seguido, comprova que a avaliação otorrinolaringológica, a fibrobroncoscopia e o RX de tórax são exames indispensáveis no paciente portador de CEE para o diagnóstico de tumores sincrônicos na árvore respiratória, cabeça e pescoço. O tipo histológico predominante da neoplasia associada foi o carcinoma epidermóide, presente em 13 de 19 pacientes.

Da mesma forma, julgamos imperiosa a avaliação minuciosa do tubo digestivo superior e, principalmente, do esôfago, através de endoscopia com corantes como o lugol, em pacientes com carcinoma de cabeça e pescoço. Ina et al. ${ }^{3}$ publicaram trabalho realizado na Medical and Dental University de Tóquio, no qual relataram que em 101 pacientes com câncer da cavidade oral e em 26 com neoplasia da orofaringe, submetidos a exame endoscópico do esôfago corado com lugol, encontraram em oito deles $(6,3 \%)$ carcinoma epidermóide. Esses pacientes eram clinicamente assintomáticos, e em cinco deles a lesão esofágica foi classificada como superficial.

Os relatos da literatura corroboram a nossa experiência no GCEEID-HCPA, quando encontramos 19 casos $(7,28 \%)$ de neoplasias associadas ao CEE, em 261 pacientes estudados, sendo os tumores aerodigestivos responsáveis por 13 (4,98\%). O carcinoma epidermóide foi o tipo histológico em todos esses pacientes e localizados na árvore respiratória $(53,8 \%)$, cavidade oral e or ofaringe $(23 \%)$ e laringe (23\%). Esses dados obrigam que o estadiamento do CEE inclua uma avaliação rigorosa do trato aerodigestivo, e vice-versa. No que diz respei to aos fatores de risco - tabagi smo e al cool ismo - estavam presentes em associação em 73,6\% da casuística.

Além de protocolos de estadiamento completos, o acompanhamento pós-tratamento desses pacientes com CEE ou tumores aerodigestivos implica em exame direto ou endoscópico, pelo menos anual, dos órgãos suspeitos. Marchant et al. ${ }^{1}$ demonstraram, em trabalho publicado no American J ournal of Otolaryngology, que o follow up dos pacientes com carcinoma da cabeça e pescoço realizado nos EUA pelos médicos da especialidade é incompleto. A mai oria dos especialistas consul tados realiza, no seguimento desses pacientes, apenas o exame direto da lesão primária já tratada e Rx de tórax. A endoscopia esofágica com lugol e a fibrobroncoscopia estão indicadas no foll ow up desses pacientes, pois mais de $90 \%$ del es estão ou foram expostos aos principais fatores de risco. Em nosso serviço, além do protocolo de estadiamento dos pacientes com CEE, desenvolveu-se um protocolo de seguimento que inclui a reavaliação periódica semestral do trato aerodigestivo. O objetivo é diagnosticar prováveis neoplasias metacrônicas posteriores em fase inicial, no trato aerodigestivo, associadas ao CEE.

Observaram-se, também, outras neoplasias associadas ao CEE que não são tumores aerodigestivos, mas que na série aqui estudada foram importantes. A principal delas foi o adenocarcinoma gástrico, presente em quatro casos (21\%). Na literatura, não foram encontradas referências da associação de CE E e câncer gástrico. O diagnóstico da lesão gástrica é fundamental, pois o estômago é a víscera preferida pela maioria dos cirurgiões para a reconstrução do trânsi to após esofagectomia com intenções curativas ou em cirurgias paliativas tipo by pass. Muitas vezes, o diagnóstico do tumor gástrico é dificultado pela impossibilidade de se examinar o estômago na endoscopia digestiva alta, pois a neoplasia esofágica que obstrui $2 / 3$ da luz do órgão impede a passagem do endoscópio.

\section{CONCLUSÃO}

F undamentados nos resultados encontrados neste estudo e na literatura internacional, afirmamos que o estadiamento dos pacientes tabagistas e alcoolistas com carcinoma epidermói de do esôfago deve ser realizado mediante protocol o que inclua o exame direto ou endoscópico da boca, or ofaringe e árvore respiratória, evitando desta maneira que neoplasias sincrônicas desses órgãos deixem de ser diagnosticadas. No seguimento dos pacientes com CEE, deve-se reavaliar semestralmente os órgãos do trato aerodigestivo, na busca de neoplasias metacrônicas posteriores em fase inicial. Sempre que factível, a gastroscopia deve ser realizada na procura de possíveis lesões neoplásicas simultâneas do estômago. 


\section{SUMMARY}

\section{Esophageal epidermoid cancer associated neo- plasms}

I NTRODUCTION. The esophageal epidermoid cancer has an important association with aerodigestive tract neoplasms and possibly share the same risk factors. Furthermore, other neoplasms can be associated with esophagus cancer.

ОвлестіVE. To analyze retrospectively the patients with esophageal epidermoid cancer (EEC) and associated neoplasms, treated by the Esophagus Stomach and Small Intestine Group of Surgery at Hospital de Clinicas de Porto Alegre from J anuary 1988 to J une 1995.

Patients And Methods. Nineteen (7.28\%) of the 261 studied patients had associated neoplasms to the EEC. Ten patients presented synchronic tumours and 9 metachronic ones. The predominant sex was the mascul ine with 17 cases. The mean age was 62.52 years in the moment of the esophageal cancer diagnostic.

RESULTS. The aerodigestive tumours, squamous carcinomas in totality, represented the predominant associated neoplasm histological type in $68.42 \%$ of the cases. The most frequent associated aerodigestive tumours site was the respiratory tract $(53.8 \%)$, followed by the oral cavity and oropharynx (23\%) and larynx (23\%). In our sample, twelve patients were smokers and 9 were alcohol abusers. In relation to the EEC treatment, surgery was performed in 6 patients. The associated neoplasm was treated with radical surgery in 11 patients and radiotherapy in 5 . Surprisingly 4 cases $(21 \%)$ of gastric adenocarcinoma associated to the EEC were diagnosed, treated with radical surgery in 3 patients.

CONCLUSION. The authors call attention to the importance of a criterial staging as well as the foll ow up in patients with EEC owing to the significant association with others neoplasms, principally with aerodigestive tumours, and discuss the common risk factors possibility: tobacco and alcohol use. Important association with gastric neoplasms were found in this casuistry. [Rev Ass Med Brasil 1997; 43(4): 335-9.]

KEY WORDS: Squamous cell neoplasms. Esophageal neoplasms. Multiple primary neoplasias. Associated neoplasms. Aerodigestive tumours.

\section{REFERÊNCIAS BIBLIOGRÁFICAS}

1. Marchant FE, Lowry LD, Moffit JJ, Sabbagh R. Current national trends in the posttreatment follow-up of patients with squamous cell carcinoma of the head and neck. Am J Otolaryngol 1993; 14: 88-93.

2. Savary $\mathrm{M}$, Monnier $\mathrm{Ph}, \mathrm{Pasche} \mathrm{R}$ et al. Multiple primary malignancies. Adv Otorhinolaryngol 1991; 46: 165-75.

3. Ina $\mathrm{H}$, Shibuya $\mathrm{H}$, Ohashi I. The frequency of a concomitant early esophageal cancer in male patients with oral and oropharyngeal cancer. Cancer 1994; 73: 2.038-41.

4. Paraf F, Fléjou J F, P otet F, M olas G, F ékété $F$. E sophageal squamous carcinoma in five patients with barret's esophagus. Am J Gastroenterol 87: 746-50.

5. IARC Working Group on theE valuation of Carcinogenic Risks to Humans. IARC monographs on the evaluation of carcinogenic risks to humans. Tobacco smoking, 38, Lyon, IARC, 1986.

6. IARC Working Group on the Evaluation of Carcinogenic Risks to Humans. IARC monographs on the evaluation of carcinogenic risks to humans. Alcohol drinking, 44, Lyon, IARC, 1988.

7. Morita M, Kuwano $\mathrm{H}$, Ohno $\mathrm{S}$ et al. Multiple occurrence of carcinoma in the upper aerodigestive tract associated with esophageal cancer: reference to smoking, drinking and family history. Int J Cancer 1994; 58: 207-10.

8. Li J Y, Ershow AG, Chen ZJ et al. A case-control study of cancer of the esophagus and gastric cardia in Linxian. Int J Cancer 1989; 43: 755-61.

9. Ghadirian P. Family history of esophageal cancer. Cancer 1985; 56: 2.112-6.

10. Coffin CM, Rich SS, Dehner LP. Family aggregation of nasopharyngeal carcinoma and other malignancies. Cancer 1991; 68: 1.323-8.

11. Slaughter DP, Southwick HW, Smejkal W. "Field cancerization" in oral stratified squamous epitelium. Cancer 1953; 6: 963-8.

12. Elias D, Mankarios H, Lasser Ph et al. Cancers épidermoïdes de l'oesophage opéré. Incidence élevée des néoplasies associées. Presse Méd 1992; 21: 652-6. 